\title{
Stability of Periodically Focused Intense Particle Beams
}

\author{
R. Pakter and F. B. Rizzato \\ Instituto de Física, Universidade Federal do Rio Grande do Sul, P.O. Box 15051, 91501-970, Porto Alegre, RS, Brazil
}

(Received 9 October 2000; published 9 July 2001)

\begin{abstract}
A stability analysis of periodically focused intense particle beams based on the beam envelope equation is performed. We show that (i) the scenario, as the focusing field increases, is not the existence of a single threshold above which stable matched (equilibrium) solutions are absent, as generally believed, but the existence of successive regions of stability interrupted by gaps of instability; (ii) the beam can be focused to tighter radii using new stable matched solutions found for focusing field strengths greater than the previous threshold. Self-consistent simulations validate the findings.
\end{abstract}

DOI: $10.1103 /$ PhysRevLett.87.044801

PACS numbers: 41.85.Ja, 41.75.-i

Confinement of low-emittance, high-current beams in periodically focusing systems [1-5] is crucial for the development of several advanced particle accelerator applications, from heavy-ion fusion to spallation neutron source [6], as well as applications in basic science. A key aspect of periodically focused beams is their stability properties. Previous studies based on kinetic theory [2] and on the analysis of the beam envelope [7-9] revealed that within a relatively limited range of variation of the focusing field strength, only one equilibrium solution with the beam radius displaying the same periodicity of the external confining field is present; we call this the matched solution. The matched solution was shown to present several transient instabilities as the focusing field strength is varied [2], and these instabilities were found to be closely related to nonlinear resonances involving the oscillatory frequencies of the focusing field and of the electrostatic perturbations propagating with the beam [9]. In particular, it was shown that above a certain threshold of the focusing field strength the matched solution undergoes a major bifurcation and loses stability [2,9], which creates severe limitations to the practical use of periodic focusing as a confining tool. In this paper we perform a detailed stability analysis of periodically focused intense particle beams, based on the beam envelope equation. In particular, we consider a high-current beam in a periodic solenoidal focusing field. Poincaré mappings allied to the Newton-Raphson method [10] are employed to precisely locate and determine the stability of matched solutions in the appropriate phase space. It is shown that these solutions undergo a series of direct and inverse bifurcations as the parameters of the system are varied. Particularly, it is shown that although the matched solution analyzed in previous works becomes unstable and eventually vanishes as the focusing field strength increases, stability is recovered for yet larger fields because new stable matched solutions emerge in the phase space. The general scenario as one increases the focusing field is thus not the existence of a single threshold above which confinement is impossible, but the existence of regions of stability interrupted by gaps where either the matched solution becomes unstable or is absent. Trans- verse sizes are also analyzed and we learn how to focus the beam to tighter radii using the new stable matched solutions found for focusing strengths greater than the previously established threshold.

In the paraxial approximation the dimensionless envelope equation for a beam in a periodic solenoidal focusing magnetic field reads

$$
\frac{d^{2} r_{b}}{d s^{2}}+\kappa_{z}(s) r_{b}-\frac{K}{r_{b}}-\frac{1}{r_{b}^{3}}=0 .
$$

$s=z / S=\beta_{b} c t / S$ is the coordinate along the beam axis, $r_{b}(s)=r_{b \text {,dimensional }} /(S \epsilon)^{1 / 2}$ is the beam radius, $K=$ $2 q^{2} N_{b} S / \epsilon \gamma_{b}^{3} \beta_{b}^{2} m c^{2}$ is the beam perveance, and $\kappa_{z}(s)=$ $\kappa_{z}(s+1)=q^{2} B_{z}^{2}(s) S^{2} / 4 \gamma_{b}^{2} \beta_{b}^{2} m^{2} c^{4}$ is the focusing strength parameter, where $B_{z}(s)$ is the magnetic field on the beam axis, $S$ is the periodicity length of the focusing field, $\beta_{b} c$ is the average axial velocity, $c$ is the speed of light in vacuo, $\epsilon$ is the unnormalized emittance of the beam, $N_{b}$ is the number of particles per unit axial length, and $q, m$, and $\gamma_{b}=\left(1-\beta_{b}^{2}\right)^{-1 / 2}$ are, respectively, the charge, mass, and relativistic factor of beam particles. For the sake of simplicity we consider $\kappa_{z}(s)$ in the form $\kappa_{z}(s)=\sigma_{0}^{2}[1+\delta \cos (2 \pi s)]$, with $\sigma_{0}=$ $\left[\int_{0}^{1} \kappa_{z}(s) d s\right]^{1 / 2}$ representing the vacuum phase advance in the smooth-beam approximation and with $0 \leq \delta \leq 1$ as the amplitude of the focusing field oscillations. The results to be presented here are independent of the specific form of $\kappa_{z}(s)$. We first notice that if the magnetic field is uniform with $\delta=0$ and $\kappa_{z}(s)=\sigma_{0}^{2}=$ const, the envelope equation represents an autonomous 1 degreeof-freedom Hamiltonian system which is known to be completely integrable yielding regular trajectories [10]. In this case there is one equilibrium radius $r_{b 0}=\left[2 K+2\left(K^{2}+4 \sigma_{0}^{2}\right)^{1 / 2}\right]^{1 / 2} / 2 \sigma_{0} . \quad r_{b 0}$ is dynamically stable with the wave number of small linear oscillations of wavelength $\lambda$ around the equilibrium yielded by [9]

$k_{0} \equiv 2 \pi / \lambda=\left[4 \sigma_{0}^{2}+K^{2}-K\left(4 \sigma_{0}^{2}+K^{2}\right)^{1 / 2}\right]^{1 / 2}$.

The wave number $k_{\max }$ of oscillations far away from equilibrium can also be calculated in uniform fields if for large 
$r_{b}$ 's one discards $1 / r_{b}$ and $1 / r_{b}^{3}$ in Eq. (1) but takes into account the effect of these "centrifugal" forces at $r_{b} \approx 0$; this is essentially a harmonic well blocked at $r_{b}=0$ from which one has $k_{\max }=2 \sigma_{0}$. Note that $k_{\max }>k_{0}$; this fact, not properly noticed earlier, has relevant consequences to be discussed shortly. When $\delta \neq 0$, we resort to nonlinear dynamics techniques and evaluate the stability index $\alpha$ of periodic orbits as represented on a Poincaré plot of the phase space where we record the pair $\left(r_{b}, d r_{b} / d s\right)$ at integer values of $s$ [10]. We define $\alpha=\cos \left(k_{\mathrm{fix}}\right)$, where $k_{\mathrm{fix}}$ is the wave number of small linear oscillations around the periodic trajectory (fixed point in the Poincaré plot) and is calculated numerically using a Newton-Raphson method. If $|\alpha|<1$ for $k_{\mathrm{fix}}$ real, the orbit is stable; if $\alpha$ reaches the upper boundary $\alpha=+1$ the orbit undergoes an inverse tangent bifurcation with a previous unstable fixed point, and if the lower boundary $\alpha=-1$ is crossed, the orbit undergoes a period doubling bifurcation losing stability. When $\delta=0$, one can simply evaluate $\alpha$ for the central fixed point in the form $\alpha=\cos \left(k_{0}\right), k_{0}$ from Eq. (2). In this case, the argument of the cosine function is real, $\alpha$ is always bounded by +1 and -1 and no bifurcations occur in the phase space. On the other hand, when the perturbation is turned on with $\delta \neq 0$ various bifurcations mark their presence on the phase space. These bifurcations are analyzed in Fig. 1, where we plot the stability index $\alpha$ as a function of the vacuum phase advance $\sigma_{0}$ (in degrees) for a uniform magnetic field $\delta=0.0$ (dashed line), a perturbative case with $\delta=2.0 \times 10^{-3} \ll 1$ (thin
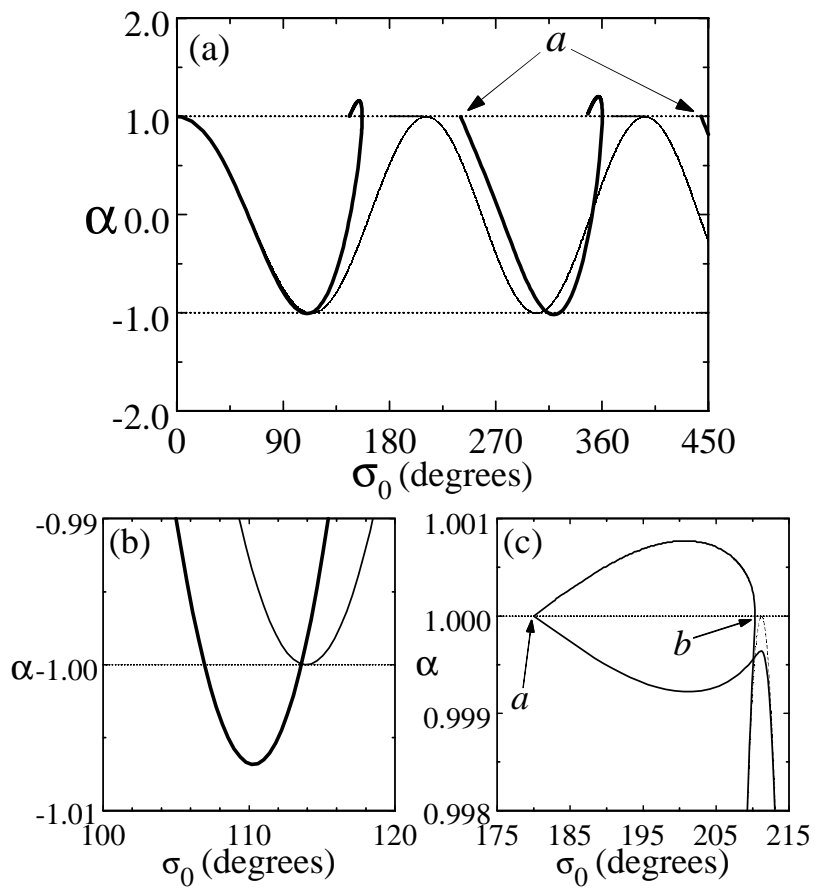

FIG. 1. Stability diagram $\alpha \times \sigma_{0}$ for $K=3.0$ and $\delta=0.0$ (dashed line), $\delta=2.0 \times 10^{-3}$ (thin solid line), and $\delta=1.0$ (thick solid line). In panels (b) and (c) we zoom in panel (a) close to bifurcations. solid line), and a nonperturbative case with $\delta=1.0$ (thick solid line). Note that the $\delta=0.0$ curve is generally not visible because the $\delta=2.0 \times 10^{-3}$ curve overshadows it for most of the values of $\sigma_{0}$; the only regions where these two curves can be distinguished are close to the bifurcations, as discussed next. First of all we observe that when $\delta \neq 0$, the first valley of the curve $\alpha$ vs $\sigma_{0}$ drops to a position slightly below the lower boundary $\alpha=-1$. This is shown in detail in Fig. 1(b), where we see that the $\delta=1.0$ matched solution clearly crosses the $\alpha=-1$ line. This means that at this point the matched solution undergoes a period doubling and becomes unstable, as noticed in a series of previous works in connection to the strong $\sigma_{0}=90^{\circ}$ instability; search for stable solutions traditionally does not go beyond this point. However, if one continues to increase $\sigma_{0}$, we see that the matched solution crosses back $\alpha=-1$, recovering stability. The gap of instability for the $\delta=1.0$ matched solution can be estimated from Fig. 1(b) as $\Delta \sigma_{0} \approx 8^{\circ}$. Although not clear from Fig 1(a), the $\delta=2.0 \times 10^{-3}$ matched solution also undergoes the same sequence of bifurcations, the difference being the size of the instability gap which is shorter: $\Delta \sigma_{0} \approx 0.016^{\circ}$. In fact, any $\delta \neq 0$ would induce the sequence of bifurcations, with smaller $\delta$ 's, generating shorter gaps. The sequence (before, during, and after period doubling) is displayed as Poincaré plots in panels (a)-(c) of Fig. 2, for $\delta=1.0$.

Perturbative cases, $\delta \ll 1$.- Remaining bifurcations in phase space are now initially examined under a perturbative regime $\delta=2.0 \times 10^{-3} \ll 1$. We focus attention on resonances with the same periodicity of the driver since those can establish new matched solutions. Resonances on phase space can be located with help of the condition $k\left(r_{b, \max }\right)=2 \pi$, where $k\left(r_{b, \max }\right)$ is the wave number of an orbit initially launched at $d r_{b} / d s=0$ and $r_{b}=r_{b, \text { max }}>r_{b 0}$; note that $k\left(r_{b 0}\right)=k_{0}$ and $k\left(r_{b, \text { max }} \rightarrow\right.$ $\infty)=k_{\max }=2 \sigma_{0}$. Suppose that initially $2 \sigma_{0}<2 \pi$. As $\sigma_{0}$ grows due to an increase of the focusing field, for instance, there will be an instant where $2 \sigma_{0}=2 \pi$; at this moment the corresponding resonance invades the phase space placing itself over the trajectory varying between the extremes $r_{b, \text { min }}=0$ and $r_{b, \max } \rightarrow \infty$. In the stability diagram $\alpha$ vs $\sigma_{0}$ the onset of the resonance is observed as a direct tangent bifurcation with the birth of two new matched solutions - one stable with $\alpha<1$ and one unstable with $\alpha>1$. The bifurcation is indicated by the letter " $a$ " in Fig. 1(c) and occurs at $2 \sigma_{0}=2 \pi\left(\sigma_{0}=\right.$ $180^{\circ}$ ) as expected. As $\sigma_{0}$ keeps growing, the resonance migrates towards $r_{b 0}$, and $k\left(r_{b, \max }\right)$ approaches $k_{0}$. In Fig. 2(d) a Poincaré plot for $\delta=2.0 \times 10^{-3}$ and $\sigma_{0}=$ $207^{\circ}$ shows the three fixed points corresponding to the original matched solution plus the newly born stable and unstable matched solutions. Further increase of $\sigma_{0}$ leads to the condition $k\left(r_{b, \max }\right)=k_{0}$ where the original fixed point undergoes an inverse tangent bifurcation with the unstable fixed point of the resonance. At this point, " $b$ " in 



FIG. 2. Poincare plots of the $r_{b} \times d r_{b} / d s$ phase space. $K=$ 3.0 and (a) $\delta=1.0, \sigma_{0}=75.4^{\circ}$; (b) $\delta=1.0, \sigma_{0}=109^{\circ}$; (c) $\delta=1.0, \sigma_{0}=135^{\circ}$; (d) $\delta=2.0 \times 10^{-3}, \sigma_{0}=207^{\circ}$; and (e) $\delta=1.0, \sigma_{0}=292^{\circ}$.

Fig. 1(c), the original matched solution vanishes. However one is still left with the stable matched solution of the resonance. As $\sigma_{0}$ increases, the stable matched solution displaces backwards toward $r_{b}=0$ in the phase space and the events of the original matched solution are reproduced: the new stable fixed point undergoes a direct followed by inverse period doubling bifurcations at $\alpha=-1$ [ $\sigma_{0} \approx 300^{\circ}$ in Fig. 1(a)], and an inverse tangent bifurcation at $\alpha=+1$ with an unstable fixed point of a yet newer resonance which invades the phase space when the new resonance condition $2 \sigma_{0}=4 \pi$ is met $\left[\sigma_{0} \approx 400^{\circ}\right.$ in Fig. 1(a)]. The newer stable matched solution associated with the $k\left(r_{b, \max }\right)=4 \pi$ resonance survives and the whole process repeats itself roughly every $180^{\circ}$ in $\sigma_{0}$, for increasingly larger values of $n$ indexing the general resonance condition $k\left(r_{b, \max }\right)=2 n \pi$. Therefore, excluding some gaps of instability with $\alpha<-1$, there is always a stable matched solution for the envelope equation which persists and can be used to confine intense particle beams to tighter radii much beyond $\sigma_{0}=180^{\circ}$.

Nonperturbative cases, $\delta \sim 1$.- For larger $\delta$ 's, the scenario described above presents some changes. We have mentioned that after the unstable fixed point of a resonance $k\left(r_{b, \max }\right)=2 n \pi$ undergoes an inverse tangent bifurcation with the original fixed point (in the case $n=1$ ) or with the stable fixed point of a previous resonance $k\left(r_{b, \max }\right)=2(n-1) \pi(n=2,3, \ldots)$, the stable fixed point corresponding to $k\left(r_{b, \max }\right)=2 n \pi$ starts moving backwards toward $r_{b}=0$. If $\delta$ is sufficiently small the stable fixed point never actually reaches $r_{b}=0$ and remains present in the phase space. However, if $\delta$ clears a certain threshold, the stable fixed point eventually reaches $r_{b}=0$ and ceases to exist. At this point, no closed orbit remains in the phase space. For $\delta=1.0$ the initial life span of the stable point is so short that it cannot be pinpointed near the tangent bifurcations of Fig. 1(a). One also sees from Fig. 1(a) that the point remains absent in wide regions along $\sigma_{0}$ (around $\sigma_{0}=200^{\circ}$ and around $\sigma_{0}=400^{\circ}$ ). These gaps are to be avoided if the goal is beam confinement. Now by further increasing $\sigma_{0}$, the system retrieves its closed orbits [10] and the stable fixed point reappears in the phase space, such as in the points marked by the letter " $a$ " in Fig. 1(a)—confinement becomes possible again. Figure 2(e) considers $\sigma_{0}=292^{\circ}$ to show how stable typically is the fixed point after its reappearance; no chaotic activity can be devised in the panel. Therefore, for large $\delta$ 's one alternates windows of stability in $\sigma_{0}$ where a stable matched solution exists, and forbidden gaps (the meaning of "forbidden" taken in the context of confinement), where the matched solution either exists but is unstable, or is simply absent; further analysis reveals that the size of the forbidden gaps increases with $\delta$.

We also perform self-consistent simulations using Green's function method [11], launching 1200 macroparticles according to a Kapchinskij-Vladimirskij (KV) distribution [1] with a beam radius corresponding to the stable matched radius. The finite number of particles in the initial condition acts as the seed for any possible instability to develop. As the beam propagates along the focusing channel we compute the $\mathrm{KV}$ beam radius $\bar{r}_{b}=\left(2\left\langle r_{j}^{2}\right\rangle\right)^{1 / 2} \equiv\left(2 r_{\mathrm{RMS}}^{2}\right)^{1 / 2}$ [9], and the amplitude of azimuthal beam modes $A_{m}=\left|\left\langle e^{i m \phi_{j}}\right\rangle\right|, m=0,1, \ldots$, where $r_{j}$ and $\phi_{j}$ are the cylindrical coordinates of the $j$ th macroparticle and $\langle\cdots\rangle$ represents the average over macroparticles. The results of a typical run over 100 periods for the case analyzed in Fig. 2(e) are shown in Figs. 3(a)-3(c). The figures reveal that beam transport is well behaved with neither radial [Fig. 3(a)] nor azimuthal [Fig. 3(b)] instabilities affecting the periodicity of a symmetrical $m=0$ beam shape. In addition, and importantly, no emittance growth was detected so beam quality is preserved. In Fig. 3(c) we display the last four periods of the run of Fig. 3(a) to show the good agreement between the beam envelope obtained from Eq. (1) (solid line) and from the self-consistent simulation (circles).

A figure of merit, if one is interested in beams with the smallest possible transverse dimensions, is the minimum value attained by the matched and stable beam envelope 

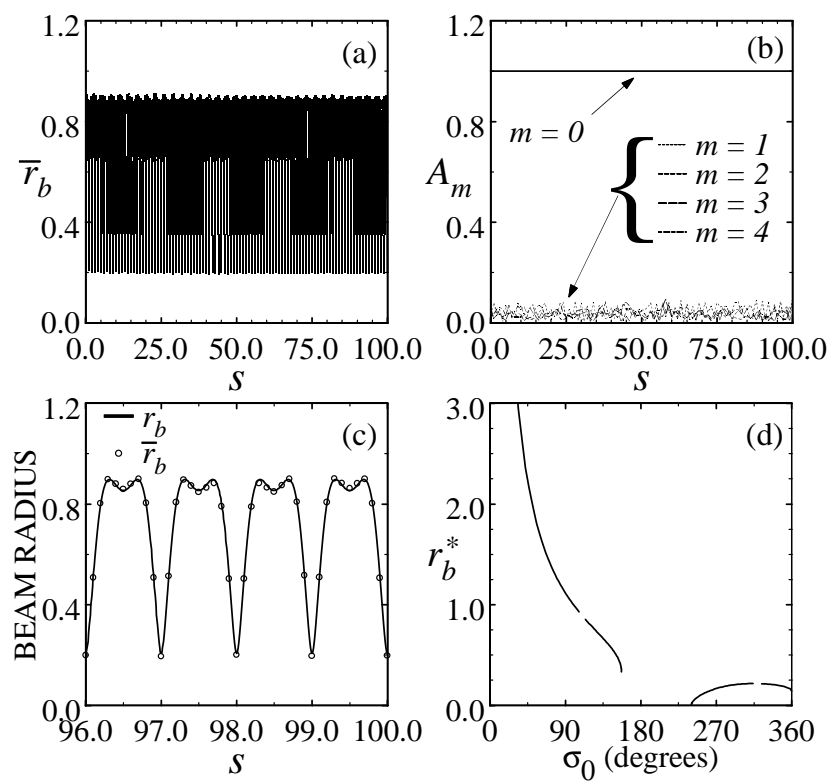

FIG. 3. Self-consistent simulations for (a) $\bar{r}_{b} \times s$ and (b) $A_{m} \times s$, for the same parameters as in Fig. 2(e); (c) last four periods of panel (a) comparing $r_{b}$ and $\bar{r}_{b}$; (d) $r_{b}^{*} \times \sigma_{0}$ for $K=3.0$ and $\delta=1.0$.

as it oscillates along $s$. A final analysis is thus in order on the minimum oscillatory value of $r_{b}$, let us call it $r_{b}^{*}$, versus the strength of the focusing magnetic field $\sigma_{0}$ and is shown in Fig. 3(d). We take $\delta=1.0$ and compare $r_{b}^{*}$ of the original fixed point with that of the stable fixed point of the $k\left(r_{b, \max }\right)=2 \pi$ resonance. The most useful ranges in both cases are the ones preceding the respective period doublings [small gaps in Fig. 3(d)]. Even though stability is recovered, the fixed points become closely surrounded by an increasing number of higher-order resonances, such as in Fig. 2(c), which may overlap leading to chaos [9]. From Fig. 3(d), $r_{b}^{*}$ corresponding to $k\left(r_{b, \max }\right)=2 \pi$ is appreciably smaller than the one corresponding to the original fixed point. To perform a quantitative comparison, we take two points - one corresponding to the original fixed point and one to the resonance stable fixed point - both preceding the respective period doublings and with similar stability (same $\alpha$ ). We take the points $\sigma_{0}=80.0^{\circ}$ and $\sigma_{0}=292^{\circ}$, both with $\alpha=-0.56$ [see Fig. 1(a)], the latter corresponding to the case displayed in Figs. 3(a)-3(c). As one moves from one point to the other, the magnetic field, which is proportional to $\sigma_{0}$, increases 3.65 times, whereas $r_{b}^{*}$ decreases 6.0 times, as seen in Fig. 3(d); i.e., the decrease in $r_{b}^{*}$ is almost twice the increase in $\sigma_{0}$ if one uses the resonance stable matched solution instead of the original matched solution. In other words, an increase of the magnetic field within its own order of magnitude produces a noticeable reduction on the minimum oscilla- tory radius of the stable matched solution. Presumably, $r_{b}^{*}$ becomes even smaller as one further increases the magnetic field and moves to stable fixed points of resonances $k\left(r_{b, \max }\right)=2 n \pi$ with larger values of $n$ [12].

In summary, we have studied the stability of periodically focused intense particle beams based on the beam envelope equation. The matched solutions undergo a series of direct and inverse bifurcations as the parameters are varied, and although the original matched solution analyzed in previous works becomes unstable and eventually vanishes as the focusing field strength increases, stability is recovered for yet larger fields because new stable matched solutions emerge. Results were validated with self-consistent simulations. Thus, as the focusing field increases we do not find one single threshold above which confinement is impossible, as believed so far, but the existence of regions of stability interrupted by gaps where the matched solution either becomes unstable or absent. Tighter focused radii are shown to exist beyond previous thresholds using the new stable matched solutions.

This work was supported by CNPq, Brazil.

[1] I. M. Kapchinskij and V. V. Vladimirskij, in Proceedings of the International Conference on High Energy Accelerators (CERN, Geneva, 1959), p. 274.

[2] I. Hofmann, L. J. Laslett, L. Smith, and I. Haber, Part. Accel. 13, 145 (1983).

[3] R. C. Davidson, Physics of Nonneutral Plasmas (AddisonWesley, Reading, MA, 1990).

[4] M. Reiser, Theory and Design of Charged-Particle Beams (Wiley, New York, 1994).

[5] C. Chen, R. Pakter, and R. C. Davidson, Phys. Rev. Lett. 79, 225 (1997).

[6] See, e.g., Space Charge Dominated Beams and Applications of High-Brightness Beams, edited by S. Y. Lee, AIP Conf. Proc. No. 377 (AIP, New York, 1996).

[7] P. M. Lapostolle, IEEE Trans. Nucl. Sci. 18, 1101 (1971); F. J. Sacherer, IEEE Trans. Nucl. Sci. 18, 1105 (1971).

[8] C. J. Struckmeier and M. Reiser, Part. Accel. 14, 227 (1984).

[9] C. Chen and R. C. Davidson, Phys. Rev. Lett. 72, 2195 (1994); Phys. Rev. E 49, 5679 (1994).

[10] A. J. Lichtenberg and M.A. Lieberman, Regular and Stochastic Motion (Springer-Verlag, New York, 1992); R. Pakter, G. Corso, T.S. Caetano, D. Dillenburg, and F. B. Rizzato, Phys. Plasmas 1, 4099 (1994); J. Guckenheimer and P. Holmes, Nonlinear Oscillations, Dynamical Systems, and Bifurcations of Vector Fields (Springer-Verlag, New York, 1990).

[11] R. Pakter and C. Chen, Phys. Rev. E 62, 2789 (2000).

[12] We also found that bifurcations for other forms of $\kappa_{z}(s)$ have the same qualitative behavior, and that even for larger values of $K$ such as $K=100$, the new stability zones, although narrowing, are still present. 